\title{
Risk and opportunity management: an approach for the mechanical testing field
}

\author{
Abdul-Karim Jamal-Eddine ${ }^{1 *}$ \\ ${ }^{1}$ L2EC, 4 Rue des Frères Montgolfier, 95420 Magny-en-Vexin, France
}

\begin{abstract}
Risk and opportunity management is a rising topic in the mechanical testing field. The importance of this issue was recently reflected in the newest version of ISO 17025 which emphasized requirements for risk assessment and opportunity taking. This article investigates the most important elements regarding the topic of risk and opportunity management in an environmental testing lab, considering a real case study of the "L2EC" laboratory. Taking the multiple areas of experimental work (climatic, vibration, mechanical, etc...), the study covers multiple areas of management and quality through a comprehensive quantitative framework. Elements of risk and uncertainty control, project planning, decision making and opportunity management are eventually translated into an applicable and coherent general road plan that fulfils and integrates well in the daily framework and strategy of the laboratory.
\end{abstract}

\section{Introduction}

Risk and opportunity assessment are new topics in the ISO 17025(2017) norm [1] and therefore new for the quality management in mechanical testing labs, however these topics are not particularly new to the engineering management fields. The present work offers a preliminary, simple and general quantitative method addressing the issue of risks and opportunities. This work is founded on a real case-study of "L2EC" (Laboratoire d'Essais en Environnements Climatiques), an environmental testing lab covering a wide range of climatic, vibration and mechanical testing services based in Magny-en-Vexin near Paris, France. The aim is to offer a starting point without complicating the mathematical formulation or discussing the theoretical and philosophical background, for a method that can be refined and reinforced with the lessons learned from different players.

\section{Risk and opportunities in the testing lab}

Risk as well as opportunities can arise from different factors and in different contexts. The most important elements of project management will be applied in this analysis -which is quite relevant for the analysis of the functioning of a mechanical testing lab as experienced in "L2EC" lab. As in project management, the lab risk management centres around three important issues: cost/market, schedule and technical performance [2-3].

*Corresponding author: abdul-karim.jamal-eddine@12ec.fr ; jamaleddine.ak@gmail.com 
The cost/market problem as in any sector of industry relates to the pressure imposed by the need to make profitable returns while respecting the market supply and demand.

Schedule issues are connected to the cost/market problem since it is related to the capacity of the lab to assimilate the demands but also depends heavily on the internal organisation and the influence of some external factors.

The third important element is the technical performance of the lab, which has to guarantee the good quality of working whether it is on the human expertise side or on the resources side.

Different components of risk, namely those adopted in this paper cost, schedule and technical can be sub-structured into smaller elements. Since the aim of this paper is to present a quantitative method and not to go deeply in the qualitative analysis of risks and opportunities, only some of the most relevant examples from the experience will be presented.

From the cost/market point of view risks can be: the continuous rise in the maintenance costs and the decrease of demand on a very particular test. Two examples of schedule risk are highlighted: the overload of the schedule and thus the absence of manoeuvring margins on one hand, and the persistent delays in the clients' projects imposing delays in the lab's schedule on the other hand. Technical risks can vary from a machine breakdown, tests unconformity or the limitation of a particular resource.

Meanwhile, it is not unusual to claim that no risk comes without an opportunity, at least to some degree. From the cost point of view, an urgent demand from a client is an opportunity for a good margin of financial gain; a price concession may help securing a new client and so forth. For the schedule, a delay from a client "A" may open the window for a more profitable test project from another client " $B$ " or the acceleration of the project of client " $B$ " while not bearing the responsibility of the project's delay of client "A". For the technical component, opportunities seem rare particularly when working in a highly regulated and normalised domain; however demands for special tests constitute an opportunity if the engineers have the relevant strong technical background to deal with such demands.

Opportunities therefore can be sub-structured; however their distribution and variation by components varies widely in comparison with risks. For cost and schedule components, opportunities can be numerous while from technical point of view they tend to be limited.

\section{Quantitative approach}

First the definition of the risk will follow a definition connected to FMECA [4] (Failure mode, effects, and criticality analysis) defined as shown in equation (1):

$$
R=F \times S \times D_{r} \times p
$$

Where $R$ represents the risk, $F$ the frequency of the risk, $S$ is the severity, $D_{r}$ the detectability and $p$ is the capacity to control the risk. The factor $p$ is a very important element that needs to be always considered and refined since ISO 17025(2017) [1] emphasizes the importance of reacting to risk and corrective actions. $F, S$ and $D_{r}$ will be confined on a scale of $[1 ; b]$ where $b$ is a real number defined by the manager indicating the highest level of criticality (very frequent for $F$, very serious for $S$ and impossible to detect 
for $D_{r}$ ) depending on the margin of flexibility he is seeking for his analysis. For instance, $b$ could be equal to three, five or ten. If $b$ is equal to 10 , more subdivisions for risk levels will be feasible and therefore more flexibility in comparison with a value of 3 for $b$. While $p$ is to be defined between 0 and 1 with the increment decided by the manager, where 0 indicates a very efficient control eliminating the risk and 1 indicates a weak control of risk/corrective action that has no influence on the criticality of the situation.

In the same manner, opportunity will be defined in this paper in a similar equation:

$$
O=F \times I \times D_{o} \times c
$$

Where $O$ is the opportunity, $F$ is the frequency, $I$ is the impact and $D_{o}$ is the detectability and $c$ is a factor related to the decision making skill of the manager. $F, I$ and $D$ should be on the same scale $[1 ; b]$ used for equation (1) (for $D_{o}, b$ indicates a high detectability inversely to the detectability in the case of risk) and $c$ on the same scale used for $r$ from equation (1) (for $c$ a value 1 indicates a very strong decision making and opportunity taking and viceversa).

While these two equations are the fundamental equations, they need to be applied relevantly in order to study in a realistic manner the risk and opportunity.

For this reason, the risks and the opportunities will be divided into three different levels as shown in Fig.1: General level, component level and elementary level.

A group of elementary risk constitutes the component risk and the sum of the components risk describes the general risk. The same description applies to opportunities. Elements described in the previous section like the overload of the schedule, delays of the client's project and machine breakdown for risks for instance fall in the elementary level.

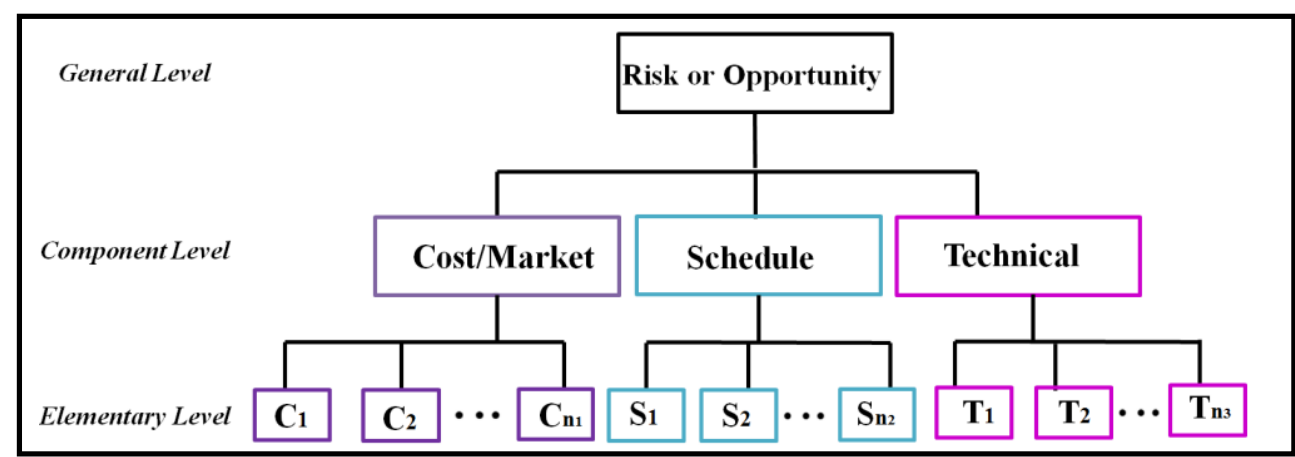

Fig. 1. Sub-structuring of risk and/or opportunities for the three main components of the problem

The component values are then calculated as the average of the corresponding elementary values as shown in equations:

$$
\begin{aligned}
R_{(C O S T)} & =\frac{\sum_{i=1}^{n_{1}} C_{i}}{n_{1}} \\
R_{(S C H E D U L E)} & =\frac{\sum_{i=1}^{n_{2}} S_{i}}{n_{2}}
\end{aligned}
$$




$$
R_{(T E C H N I C A L)}=\frac{\sum_{i=1}^{n_{3}} T_{i}}{n_{3}}
$$

Where $R$ represents the risk value while $n_{1}, n_{2}$ and $n_{3}$ will represent the number of elements chosen by the manager for cost, schedule and technical components respectively. Opportunities will be defined in the same manner for the three components $O_{(\operatorname{COST})}$, $O_{(\text {SCHEDULE) }}$ and $O_{(\text {TECHNICAL) }}$

Values at the elementary level ( $C_{i}$ or $S_{i}$ for example) will be calculated using equation (1) and equation (2) for risks and opportunities respectively.

The strategy of the lab will then be decided on the component level through two steps. First, the definition of a new concept of a "dynamic" or "adjusted" risk factor which will be defined as the difference between risk and opportunities, or in other words the risk adjusted by the opportunities that may arise around the same component:

$$
(\text { Adjusted Risk) })_{j}=A R_{j}=R_{j}-O_{j}
$$

Where $j$ represents the component which will be COST, SCHEDULE or TECHNICAL. $A R_{\text {Technical }}$ for example will tend to be empirically larger than $A R_{\text {cost }}$ since as discussed earlier in this paper; opportunities in the technical components are usually rare in comparison with the other two components.

And then adjusted factors will be applied to deduce the general level but with the possibility of "weighting" the three components in order to define the lab's strategy through adding a weight factor $k$ as follows:

$$
R_{G}=\frac{\sum_{j=1}^{m}\left[k_{j} .\left(R_{j}-O_{j}\right)\right]}{m}
$$

Where $R_{G}$ is the general adjusted risk with $j$ representing the components equal to three in this model (cost, schedule and technical). Larger $R_{G}$ values represent higher risks with smaller opportunities and vice-versa. $R_{G}$ tends to be a positive value since opportunities tend to be quantitatively smaller than risks. Furthermore and in order to control the balance between the components and in order keep the scale in coherence with scale's definition for previous elements, it is preferable that equation (7) respects the condition represented in equation (8):

$$
\left(\sum_{j=1}^{m} k_{j}\right)=m
$$


If a lab wishes to focus strongly on marketing issues and enlarging the number of his clients, the manager can increase the value of $k_{l}$ associated with the cost/market component for example. If the lab finds itself in a continuous overburden on the schedule, the manager can increase $k_{2}$ which is the weighting factor for schedule. If the lab manager sees a particular threat on the long term technical performance, he can increase $k_{3}$ assigned to technical performance and so forth. Following equation (8), any increase in a particular weighted factor $k$ will be associated with a decrease in another. Leaving too much flexibility for the weighted factors will depress the importance of factors such as frequency, detectability, severity and others defined in the first two equations and therefore the equilibrium of the model will be lost in favour of the strategical ingredient.

Following the lab's strategy, the same distribution of adjusted risk on the component level can be reflected into a different value of general adjusted risk on the general level as shown in the example of Fig.2 (using a normalised scale for a pre-calculated example on the elementary level). In Fig.2 (a), the lab follows a balanced strategy between the three components (no particular weighting); the adjusted value of risk is at a value of 3 . The same lab, for the sake of the argument, and after buying new material and machines, feels the necessity for finding new clients in order to make profit and reduce the liabilities on one hand but is aware of the absence of enough technical know-how on the short term on the other hand. Fig.2 (b) shows how a change in the strategy aiming to focus on the cost $/$ market component and the technical component (increasing $k_{1}$ and $k_{3}$ ) will impact the general adjusted risk in the lab to a value of 4 (the presence of a large number of machines reduces normally the risk while increasing the opportunities on the schedule component).

(a)


(b)
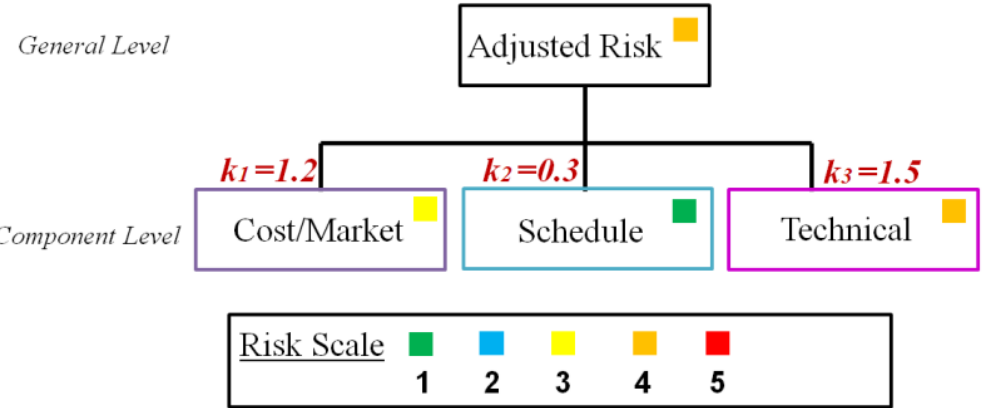

Fig. 2. (a) Component level and general adjusted risk for a perfectly balanced strategy; (b) Component level and general adjusted risk for a weighted strategy of the same example 


\section{Conclusion}

The approach offered in this paper aims not only to assess the risk but also to push forward the idea of the impact of opportunities and their management on the overall risk in the system. It provides for practitioners and lab managers, through a decentralized surveillance system, a rather robust tool of minimizing risk and increasing opportunities while respecting the quality equilibrium (technical, client satisfaction, scheduling and planning, etc...).

The present model is a simple and practical model that covers the elements necessitated for practitioners by ISO 17025 (2017) [1] but is however a foundation for further development. The presented method is a dynamic method that needs to be calibrated continuously through the development of the lab and the experience of the manager (calibrating uncertainties, frequencies, detectability, corrective actions, strategical priorities, management skills, decision making, etc.).

While opportunities and risks are studied in this paper through associating them in a qualitative manner and with a simple mathematical relationship, the non-linear and the endogenous relationships between these two elements are not detailed or studied. Future work will need to examine relationship between opportunities and risks not only through a component by component approach but also in a trans-component approach.

The author wishes to acknowledge the continuous support of Mrs. Sophie Maurin.

\section{References}

1. ISO/IEC 17025, General requirements for the competence of testing and calibration laboratories. (International Organization for Standardization, Geneva, 2017)

2. J. R. Meredith, S. J. Mantel, Project Management: A Managerial Approach, 8th ed. (John Wiley \& Sons, New York, 2011).

3. PMI, A Guide to the Project Management Body of Knowledge, 5th ed. (Project Management Institute, Pennsylvania, 2013).

4. Department of Defense U.S. Procedures for performing a failure mode, effects and criticality analysis, (Standard No. MIL-STD-1629A, Washington, DC, 1980). 